\title{
Identification of Pavement Distress Types and Pavement Condition Evaluation Based on Network Level Inspection for Jazan City Road Network
}

\author{
M Mubaraki \\ Department of Civil Engineering, College of Engineering, The University of Jazan, PO Box 706, Saudi Arabia
}

Received 30 December 2012; accepted 26 September 2013

\begin{abstract}
The first step in establishing a pavement management system (PMS) is road network identification. An important feature of a PMS is the ability to determine the current condition of a road network and predict its future condition. Pavement condition evaluation may involve structure, roughness, surface distress, and safety evaluation. In this study, a pavement distress condition rating procedure was used to achieve the objectives of this study. The main objectives of this study were to identify the common types of distress that exist on the Jazan road network (JRN), either on main roads or secondary roads, and to evaluate the pavement condition based on network level inspection. The study was conducted by collecting pavement distress types from 227 sample units on main roads and 500 sample units from secondary roads. Data were examined through analysis of common types of distress identified in both main and secondary roads. Through these data, pavement condition index (PCI) for each sample unit was then calculated. Through these calculations, average PCIs for the main and secondary roads were determined. Results indicated that the most common pavement distress types on main roads were patching and utility cut patching, longitudinal and transverse cracking, polished aggregate, weathering and raveling, and alligator cracking. The most common pavement distress types on secondary roads were weathering and raveling, patching and utility cut patching, longitudinal and transverse cracking, potholes, and alligator cracking. The results also indicated that $65 \%$ of Jazan's main road network has an average pavement condition rating of very good while only $30 \%$ of Jazan's secondary roads network has an average pavement condition.
\end{abstract}

Keywords: Pavements, Maintenance, Rehabilitation, Distress types, PCI.

$$
\begin{aligned}
& \text { تحديد أنواع عيوب رصف شدة الرصيف وتقييم حالة الرصف اعتمادا على فحص مستوى الشبكة لطرق } \\
& \text { مدينة جازان } \\
& \text { محمد مباركي } \\
& \text { الملخص: الخطوة الأولى لإنشاء نظام إدارة رصف هو تحديد شبكة الطرق ـ سمة هامة لهذا النظام هي القدرة على تحديد الوضع الحالي لشبكة الطريق }
\end{aligned}
$$

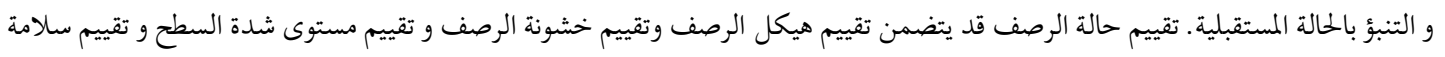

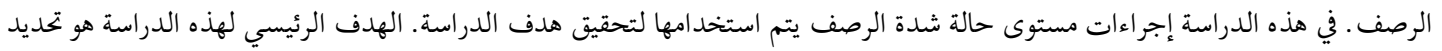

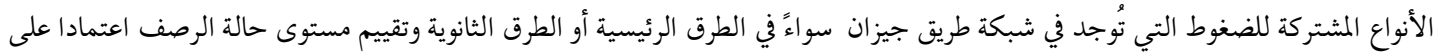

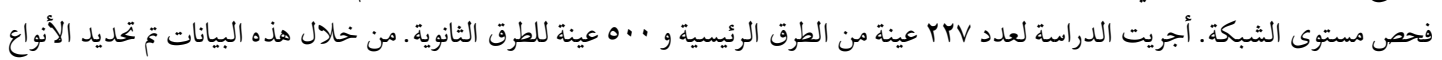

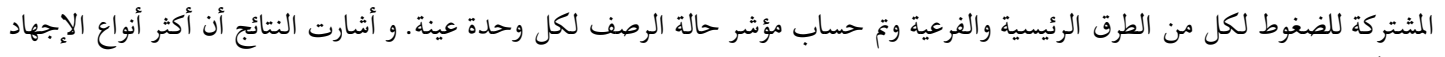

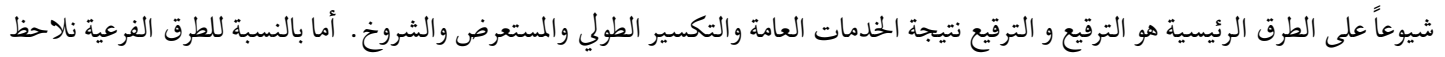

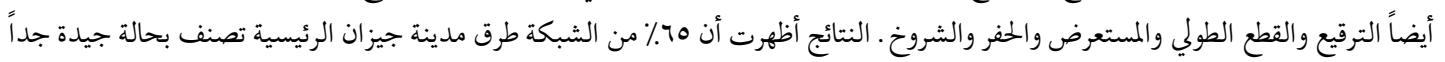

$$
\begin{aligned}
& \text { بينما ·苂 من الطرق الفرعية بحالة متوسطة. }
\end{aligned}
$$$$
\text { الكلمات المفتاحية: الرصف ، الصيانة ،التأهيل ، أنواع الإجهادات ، مؤشر حالة الرصف. }
$$

$\overline{\text { *Corresponding author's e-mail: mmubaraki@jazanu.edu.sa }}$ 


\section{Introduction}

The City of Jazan, in Saudi Arabia, has rapidly developed during the last 10 years. City pavement requires maintenance because of the impact from traffic, cuts in the roadway by utilities, and climatic conditions (Al-Swailmi 1994). Therefore, evaluation of pavement condition is a vital process for municipalities to preserve capital infrastructure and maximize its benefits. Generally, pavement condition evaluation may involve the evaluation of structural pavement, roughness, surface distress, and safety or skid resistance.

Pavement engineers have long recognized the importance of distress information in quantifying pavement quality. This information has been used to document present pavement conditions, chart past performance history, and predict future pavement condition (Shahin 2005). Pavement distress information is also broadly used as the only quality measure in many pavement management systems (PMS). This is particularly true for systems used by municipalities in urban areas where measurements of roughness and skid resistance are not performed because of lack of relative applicability (Mubaraki 2004).

On the other hand, the interrelationship between structural and functional evaluation for the pavement can be considered because there is no direct relationship. Therefore, both functional and structural evaluations of pavements are important and are meant to supplement rather than replace one other. An observation of functionality below the acceptable level is one way to trigger a structural evaluation. The rough pavement may be structurally sound and require only a level up surface layer, or it may be structurally weak and require replacement or a thick overlay (Haas et al. 1994).

Pavement distresses are visible imperfections on the surface of pavements. There are symptoms of the deterioration of pavement structures. Most if not all agencies have implemented PMS and collect periodic surface distress information on their pavement through distress surveys (Haas et al. 1994).

Surface distress evaluation is the most prevalent way to evaluate pavement condition in Saudi Arabia major cities of Riyadh, Dammam, Madinah alMunawarah, and the holy city of Makkah (Mubaraki 2010). The officials of Jazan Municipality are very anxious to upgrade the existing pavement practices to rational, systematic, and practical ones. Research has been started by collecting information on the network in order to build a PMS. This paper concerns identifying pavement distress types for the entire network. Each distress, in almost all distress evaluation methodologies, is specified by severity level (low, medium, high) and an extent level described in measurable units (linear or area). A distress type, and levels of severitty and extent are assigned a deducted value, which is an indication of how this combination, when present, affects the perfect pavement. However, road agencies or municipalities do differ in handling distress data, and it is very difficult to conclude that one system is better than the others. Therefore, each agency should select or develop a procedure to deal with distress data so that it suits their municipalities' needs and capabilities.

Once the distress data for a network are available, maintenance needs and budget can be determined. Each type of distress is the result of one or more variables, which provides great insight into the causes of pavement deterioration. Thus, for each pavement type, the proper pavement evaluation program and procedure should include the identification of pavement distress types, severity, and the number of distresses (Shahin 2005).

Pavement distress information is usually converted into a condition index. The condition index combines information from all of the distress types, severities, and quantities into a single number. This number can be used at the network level to define the condition state, identify when treatments are needed, rank or prioritize, and to derive the number used to forecast pavement condition. The condition index may represent a single distress such as fatigue cracking or a combination of many pavement distresses which is then usually referred to as a composite index. Additional information has also been included in some indices such as traffic levels and highway class to produce priority ranking indices.

The U.S. Army Corps of Engineers developed a very complete condition index for a pavement management system in 1976 (Baladi and Snyuder 1992). This includes pavement condition survey procedures and a detailed method for calculating a pavement condition index (PCI), which is still used today by many agencies. The computational procedures for this index will be shown later in this module.

Condition indices are used in most pavement management systems for the following four basic reasons (Deighton and Sztraka 1995), in order to trigger treatments, calculate life-cycle costs, evaluate the network condition, and make use of the same relative scale between systems.

The main objective of this paper is to identify pavement distress types for the entire Jazan network using the PAVER procedure and, as a result of that, investigating the network pavement condition. To achieve these objectives, the study involves sampling and data collection, identifying and selecting common types of distress, and evaluating pavement conditions. 


\section{Pavement Condition}

\subsection{Introduction}

It is common for agencies to describe pavement distress in terms of severity and extent. Severity indicates how bad the distress is while extent indicates the quantity of distress. Extent can be estimated for an entire section length or over a representative area (such as 100 meters per km), or measured. Together, these two parameters can describe a great deal about a particular distress.

To transform this data into a meaningful condition index, deduct values are needed. Deduct values are points which are used to compute the index based on the severity and extent of the distress represented. The development and calibration of the proper deduct value is the most complicated and critical part in the development of a PCI.

The index base is calculated by deducting a number of points from the index value of a section of pavement in perfect condition depending on the severity and extent of the deficiency. The value deducted also depends on the condition of the pavement. The number of points deducted is called the deduct value.

The deduct values should be scaled such that the resulting condition index threshold value (or action point) occurs at about the middle of the scale (Deighton and Sztraka 1995). In the past, some have established the "should consider action" level at about $60 \%$ of the scale and the "must consider action" level at $40 \%$ of the scale. Moreover, the transition of the deduct values through the various levels of the distress matrix should produce a condition index that transitions as smoothly as possible with time.

Pavement distress is usually observed in the field as a continuous process with time, as the distress progresses through the full range of severity and extent. The trends of the PCI in the PMS should correspond to the trends observed in the specific pavement section represented by that index. Most deficiencies, once they become apparent, tend to increase in both severity and extent at an increasing rate with time. Thus, the PCI which is the numeric representation of the pavement condition in the field, should have the same trends with time as the deficiency appears to have in the field. In general, those pavements that deteriorate rapidly after the last treatment tend to have a fairly linear form (ie. the rate of change in pavement condition is about the same from one year to the next). The pavement that lasts longer before some distress is observed tends to be more exponential in form. Pavements that have lasted an unusually long time before distress occurs tend to deteriorate quite rapidly in the end; thus, they appear to have a very sharp exponential trend.

The very simple formula which uses a deduct value to compute a distress index is shown in the following equation:

$$
\mathrm{PCI}_{\mathrm{i}}=\mathrm{PCI}_{\max }-\text { Deduct }
$$

Where

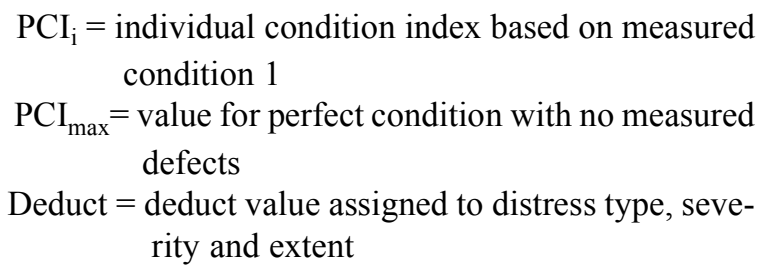

Obviously, using this equation implies that the condition index gets worse as the deduct value increases. Assume an agency uses an index with a scale from 0 (bad) to 100 ( perfect $=\mathrm{D}_{\text {max,i }}$ ). If the pavement was in perfect condition, the deduct value would be 0 , resulting in an index value of 100 . If the pavement was in terrible condition, the deduct value would be 100 , resulting in an index value of 0 .

The relative value of the pavement distress index which represents the condition of the pavement and the shape of the resulting pavement deterioration depends entirely upon the development of the deduct values. Two basic approaches are often used to develop deduct value: expert opinion, and engineering or mathematical input.

ASTM Standard D5340 provides a set of deduct value versus extent curves based on work done by Baladi and Snyder (1992). The $x$-axis represents the density or extent, while the $y$-axis represents the deduct values for each distress individually. Whatever method is chosen to assign deduct values for the individual condition indices is up to each agency. Each method has its strengths and weaknesses, and, each method can produce a different shape in the performance curve.

\subsection{Background of PAVER Development}

The development of PAVER was begun in 1974 by the Army Corps of Engineers through funding from the military services to manage the vast inventory of pavement in the U.S. Department of Defense (DoD). The intent was to build a pavement database. In 1976, the engineers developed a very important application with the help of technology-a PCI for airfield pavement. The PCI for roads and parking lots was developed in 1979. The PCI, inventory database, and basic reporting capabilities constituted the first version of PAVER, and an official prototype evaluation test was conducted at Fort Eustis, Virginia, USA. In the early 1980s, the American Public Works Association (APWA) adopted the system for use by cities and counties (Shahin and Walteher 1990).

After development, users in the field began to ask for the ability to analyze the consequence of different budgets on future pavement condition and the backlog 
of deferred major maintenance and rehabilitation (M\&R) projects. As result of this effort, PCI prediction models for different pavement types, namely asphalt concrete (AC) and Portland cement concrete (PCC), were developed. After over 3 years of intensive data collection and research efforts, it was concluded that there is no such thing as an accurate model that fits all pavements, no matter how many independent variables are included in the prediction models. Variables considered were load (frequency and intensity), climate (precipitation, temperature, freezing index, and solar radiation), and calculated stresses and strains in the pavement. As a result of developing a PCI, the engineers started to build a prediction engine called PAVER. The PAVER prediction engine enables users to develop condition deterioration trends for each family of uniform pavements in their agency. These trends are then used to predict pavement condition and perform the M\&R budget consequence analysis.

In the mid-1980s, the USA's Federal Aviation Agency (FAA) funded the production of desktop PAVER, and it became widely used by airports. In 1993, the American Society for Testing and Materials (ASTM) adopted the airfield pavement PCI as a standard. This PCI was followed a few years later by the adoption of the PCI for roads and parking lots. Now the PAVER system is widely used by municipalities and road agencies all over the world.

The PAVER building components included inventory, condition assessment, condition prediction, performance analysis, budget consequence, optimization (work planning), and project formulation. PAVER inventory management is based on a hierarchical structure composed of networks, branches, and sections, with the section being the smallest managed unit. This structure allows users to easily organize their inventory while providing numerous fields and levels for storing pavement data. To assess pavement condition, PAVER uses the PCI as its primary condition index.

PCI measures the pavement structural integrity and surface operational condition on a scale from 0 to 100 . It is calculated from measured pavement distress types, severities, and quantities and was developed to agree with collective judgment of experienced pavement engineers. In addition to the PCI, PAVER allows users to create other condition indices based on the PCI distresses already stored in the system.

PAVER uses the PCI, with PCI based on subjective observations. The index itself must be both objective and systematic to be of value. The PCI is calculated based on the results of a visual condition survey in which distress type, severity, and quantity are identified. Field verification of the PCI inspection method has shown that the index gives a good indication of a pavement's structural integrity and operational condi- tion. It provides a useful index of both the current condition and an indication of future performance. The degree of pavement deterioration is a function of distress type and severity, and the density of distress. For this reason, deduct values were introduced as a type of weighing factor to indicate the size of the effect that each particular distress type, severity level, and distress density combination has on pavement condition.

A PCI needs to be based on manageable road sections, a roads inventory, and a classification and rating system for road defects. In order to develop a PCI, the road network needs to be divided into manageable segments. Sections with a relatively uniform pavement structure, design and traffic volume will have similar performance characteristics. In urban settings, sections should be kept to a manageable length-typically one block long. Some road authorities limit the length to $100-150$ meters for problematic sections. Other authorities will use longer segments for roads that are consistent throughout their length.

Road sections in rural settings can be considerably longer-in some cases as much as 10 kilometres. Each road section needs a unique identification so that the PCI observations can be maintained in a database. Each road section should have a basic history attached to it including class, length, width, geometry, type and volume of traffic, pavement type (whether it is flexible, rigid, or composite), original construction date, maintenance and rehabilitation history, and current condition based on the last PCI.

\subsection{PCI Calculation Procedure}

These steps and the examples have been summarized from the Annual book of ASTM Standards (2011).

Step1: Determine deduct values

* Calculate the density percentage [density = (quantity /sample unit area) * 100]

* Determine the deduct value from the deduct value curves (given an example for alligator cracking as shown in Fig. 1)

Step 2: Determine the maximum allowable number of deducts $(\mathrm{m})$

* Determine the maximum allowable number of deducts $[\mathrm{m}=1+(9 / 98)(100-\mathrm{HDV})]$ where HDV is the highest deduct value.

* Reduce the number of deduct values to $m$. If the deduct values are fewer than $\mathrm{m}$, then all of the deduct values should be taken.

* List the deduct values in a descending order, given an example as in Table 1.

Step 3: Determine the maximum number of corrected deduct value (CDV) 


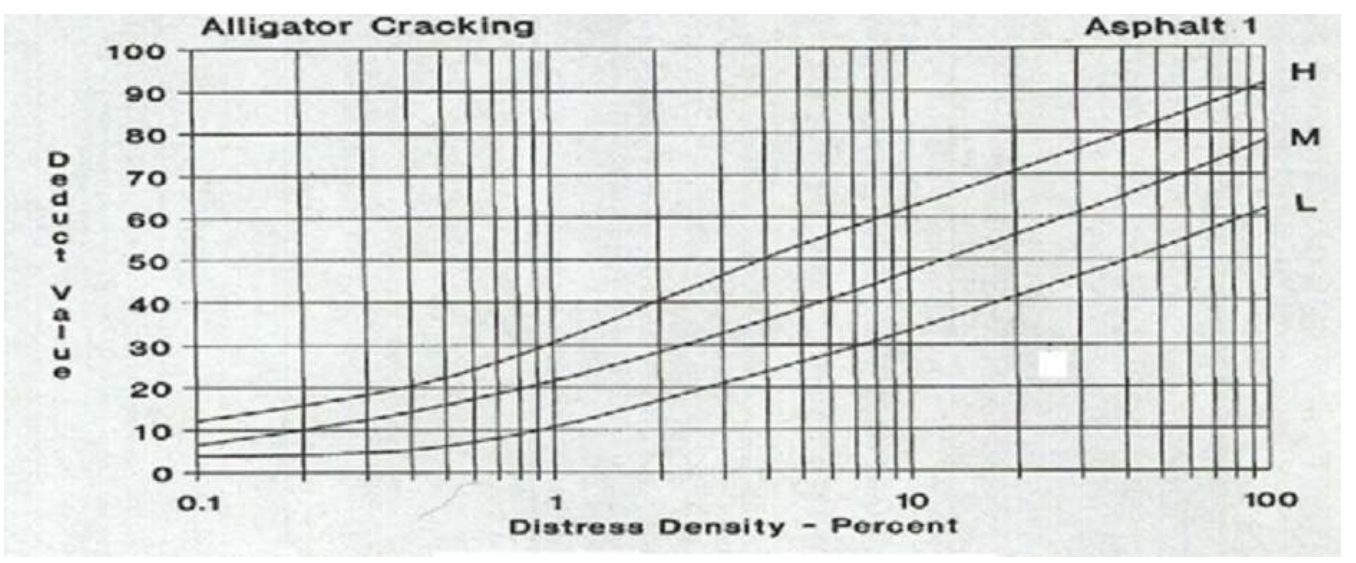

Figure 1. Deduct value for alligator cracking.

Table 1. Example of deduct values for typical road degradation.

\begin{tabular}{|c|c|c|c|c|c|c|}
\hline Distress & Code & Severity & Quantity & Density & $\begin{array}{l}\text { Deduct value } \\
\text { (from Figure } \\
\text { 1) } \\
\end{array}$ & $\begin{array}{l}\text { Deduct (maximum } \\
\text { allowable number - } \\
\text { step 2) }\end{array}$ \\
\hline \multirow{3}{*}{ Patching } & \multirow{3}{*}{11} & $\bar{L}$ & 3 & 1.875 & 3 & 32 \\
\hline & & M & 0.08 & 0.05 & 3 & 24 \\
\hline & & M & & & & \\
\hline \multirow{2}{*}{$\begin{array}{l}\text { Longitudinal and } \\
\text { transverse cracking }\end{array}$} & 10 & M & 24 & 15 & 10 & 23 \\
\hline & \multirow{3}{*}{1} & $\mathrm{~L}$ & 0.54 & 0.3375 & 4 & 21 \\
\hline \multirow{2}{*}{ Alligator cracking } & & M & 5.4 & 3.375 & 21 & 17 \\
\hline & & $\mathrm{H}$ & 2 & 1.25 & 32 & 15 \\
\hline \multirow{3}{*}{ Block cracking } & \multirow{3}{*}{3} & $\mathrm{~L}$ & 6 & 3.75 & 4 & 10 \\
\hline & & M & 1.2 & 0.75 & 2 & 4 \\
\hline & & $\mathrm{H}$ & 9.5 & 5.9375 & 23 & 4 \\
\hline \multirow[t]{2}{*}{ Jt. Ref. Cracking } & \multirow[t]{2}{*}{8} & M & 19 & 11.875 & 17 & 3 \\
\hline & & $\mathrm{L}$ & 1.35 & 0.84375 & 2 & 3 \\
\hline \multirow{2}{*}{$\begin{array}{l}\text { Reveling and } \\
\text { weathering }\end{array}$} & \multirow[t]{2}{*}{19} & M & 30.9 & 19.3125 & 24 & 2 \\
\hline & & $\mathrm{H}$ & 1.2 & 0.75 & 15 & 2 \\
\hline
\end{tabular}

* Determine the number of deducts $>2, \mathrm{q}$

* Determine the total deduct values by adding all individual deduct values

* Determine the CDV form $q$ and total deduct value for the first iteration by looking for the appropriate correction

* Set the minimum deduct value $>2$ to 2 in the next iteration and repeat the steps

* Select the maximum CDV form the calculated CDVs as the largest value

Step 4: Calculate PCI by subtracting the maximum CDV from 100

\section{Sampling and Data Collection}

Jazan Municipality divides Jazan City into 25 districts. A district is an area surrounded by four main roads, and each district covers a number of regions. A region is an area that contains a number of secondary roads surrounded by four main roads within a district. There are a total number of 110 regions in the city.

Jazan's road network is divided into two categories: main and secondary roads. Main roads represent $22 \%$ of the total network area and are defined as roads with a middle island, or with a total width of more than 30 


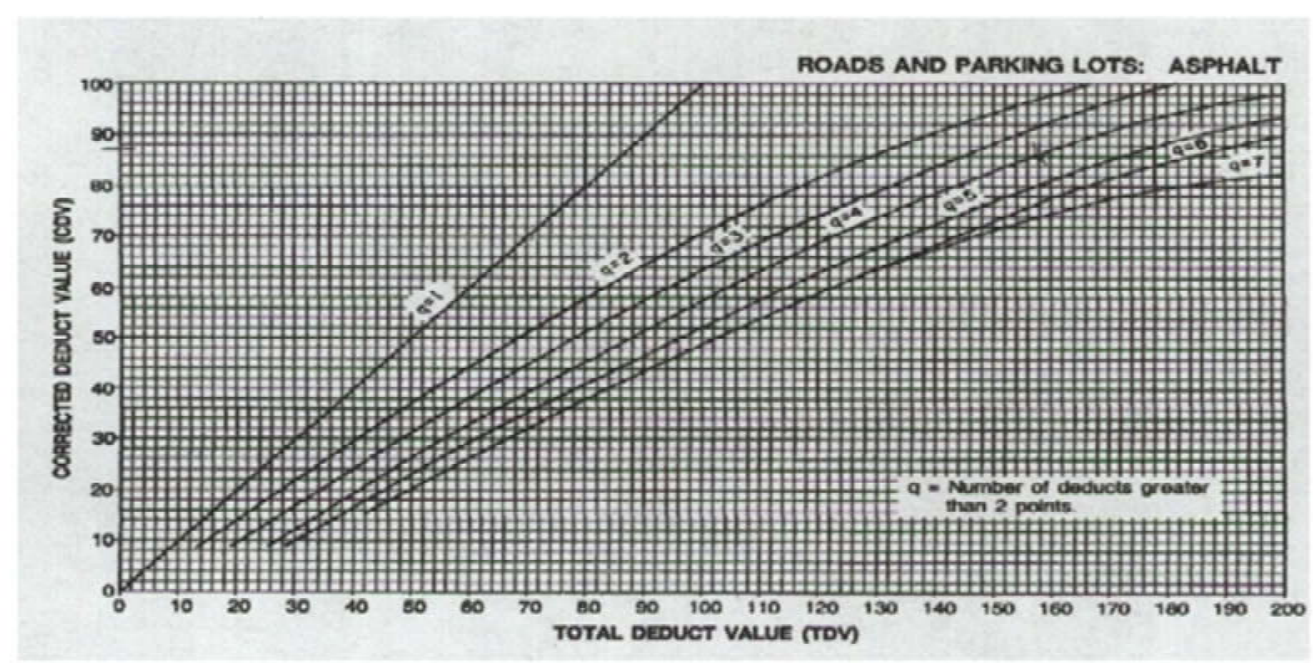

Figure 2. An example corrected deduct value.

Table 2. An example for PCI calculation.

\begin{tabular}{l|lllllllllll|l}
\hline$\#$ & \multicolumn{1}{c}{ Ded uct Values } & Total & q & CDV \\
\hline 1 & 32 & 24 & 23 & 21 & 17 & 15 & 10 & 4 & 146 & 8 & 60 \\
2 & 32 & 24 & 23 & 21 & 17 & 15 & 10 & 2 & 144 & 7 & 68 \\
3 & 32 & 24 & 23 & 21 & 17 & 15 & 2 & 2 & 136 & 6 & 66 \\
4 & 32 & 24 & 23 & 21 & 17 & 2 & 2 & 2 & 123 & 5 & 64 \\
5 & 32 & 24 & 23 & 21 & 2 & 2 & 2 & 2 & 108 & 4 & 62 \\
6 & 32 & 24 & 23 & 2 & 2 & 2 & 2 & 2 & 89 & 3 & 57 \\
7 & 32 & 24 & 2 & 2 & 2 & 2 & 2 & 2 & 68 & 2 & 50 \\
8 & 32 & 2 & 2 & 2 & 2 & 2 & 2 & 2 & 46 & 1 & 46 \\
\hline
\end{tabular}

meters without a middle island. Secondary roads account for approximately $78 \%$ of the total network area and represent the roads inside a defined region surrounded by four main roads.

Every category from the two roads is divided into branches, and the branches are divided into sections. The section for a main road is a defined distance between two intersections in the main roads. The section for a secondary road is defined as the area surrounded by four main roads. The sections for both main and secondary roads are divided into a number of sample units. A sample unit for a main road is 100 meters per lane from the section. A sample unit of the secondary main road is the distance between two intersections.

Jazan Municipality was started as a project to develop a systematic way for managing the road network. Gathering information about the city's road network and pavement condition survey were started to build a good database about the city roads in order to reach to a PMS. The survey was based on the PAVER condition survey procedure. The procedure involves determining distress types and quantities, and severity levels for each sample unit.
It is recommended that the sample units to be inspected be spaced equally throughout the section, and the first one be chosen at random. Therefore, a systematic random sampling technique was used to select sample units. This technique can be achieved by finding $(N)$ and $(n)$ where $N$ equals the total number of available sample units and $n$ equals the minimum of sample units to be surveyed, and then dividing $N$ by $n$ to get the sampling interval $(i)$. Random start $(s)$ is selected at random between sample unit 1 and $i$. The sample units to be surveyed are identified as $s, s+1, s$ $+2 i$, etc.

In this paper, the network level inspection was used to conduct the pavement condition evaluation. A network level inspection can be conducted by surveying only a few sample units per section compared to project level inspection. The paper used the following Table 1, which is based on Eqn.1 (Shahin 2005).

$$
n=\frac{N x s^{2}}{\left(\frac{e^{2}}{4}\right)(N-1)+s^{2}}
$$


Table 3. Network level sampling based on Equation 1.

\begin{tabular}{cc}
\hline No. of Sa mple Units in Section (N) & No. of Units to be inspected (n) \\
\hline 1 & 1 \\
2 to 4 & 2 \\
5 to 20 & 3 \\
Over 20 & 4 \\
\hline
\end{tabular}

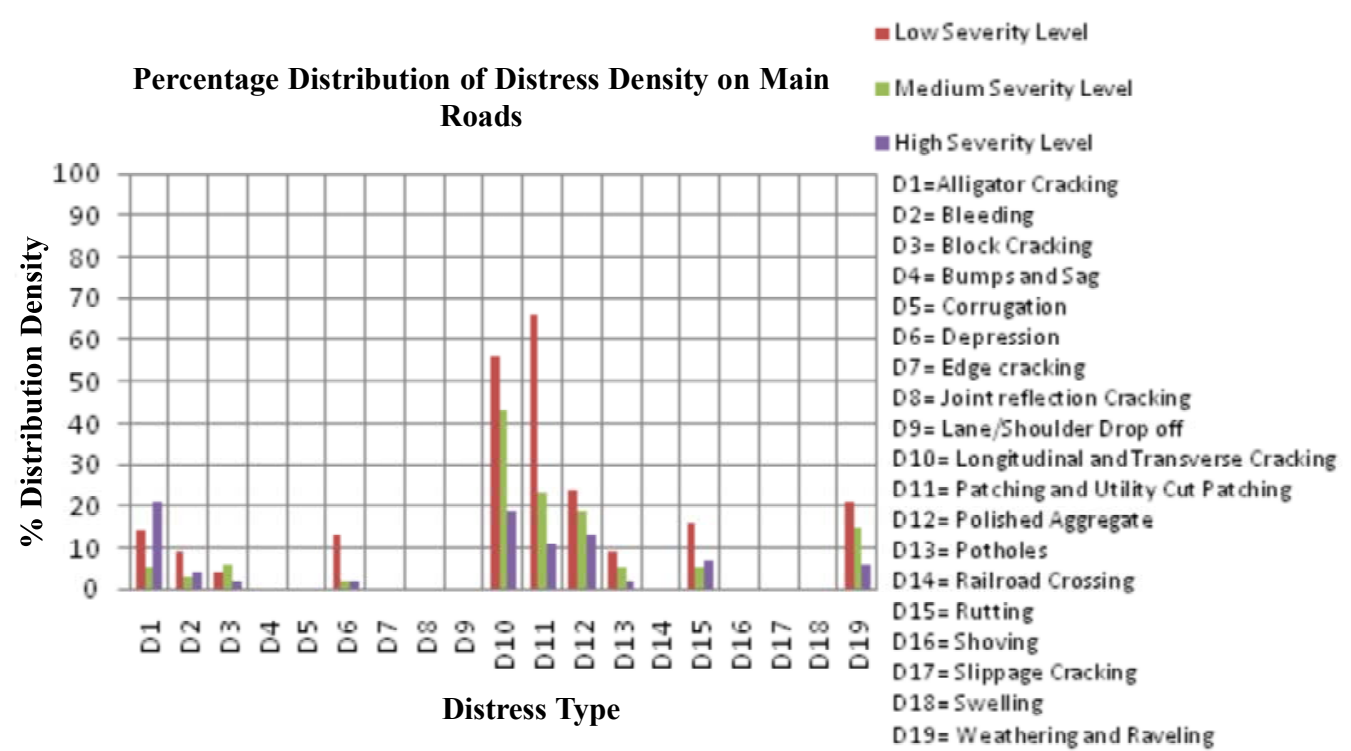

Figure 3. Percentage distribution of distress density on main road networks.

The degree of sampling presented in Table 1 is sufficient for assessing the condition of the pavement (Shahin 2005) as well as others, such as maintenance plans. Table 1 shows that the recommended values for network level inspection was based on a $95 \%$ confidence level, which means the degree of sampling taking in account in this study is reliable with an error of only $5 \%$.

Where

$N=$ total number of sample units in the pavement section

$e=$ allowable error in the estimate of the section PCI $(\mathrm{e}=5)$

$s=$ standard deviation of PCI between sample units in the section $(\mathrm{s}=5)$

This equation provides a consistent method for selecting the number of units to inspect for different size sections and this is the case for this paper's data. The rationale behind this is that management at the project level requires accurate data for the preparation of work plans and contracts. Therefore, more sample units were inspected than usually sampled for network level management. Using this number, a reasonable estimate of the true mean PCI of the section will be obtained. There is $95 \%$ confidence that the estimate is within \pm 5 points of the true mean PCI (Shahin 2005).

The condition survey was conducted by inspectors. Each inspector was sent to a particular section of the network to record the existing distress types, quantities and severity level in each selected sample unit within the section. The inspector's work supervised by an engineer to ensure work quality. Out of the 25 districts in Jazan City only 5 have pavement evaluation records so far. Out of the total 50 main roads, only 11 have pavement evaluation records so far.

Eleven main roads selected from different locations within the city were included in the analysis. Within the selected main roads, 27 sections were considered. The 27 sections were subdivided into 227 sample units with a total length of 22,270 meters. From the five districts that have data, a total of 10 regions were selected. The total numbers of sample units within these regions were 500, with a total area of 769,000 square meters.

\section{Identification and Selection of Common Types of Distress}

\subsection{Common Distress on Main Roads}

Figure 3 shows the percentage of distribution of distress types found on main road networks at three severity levels-low, medium, and high. 


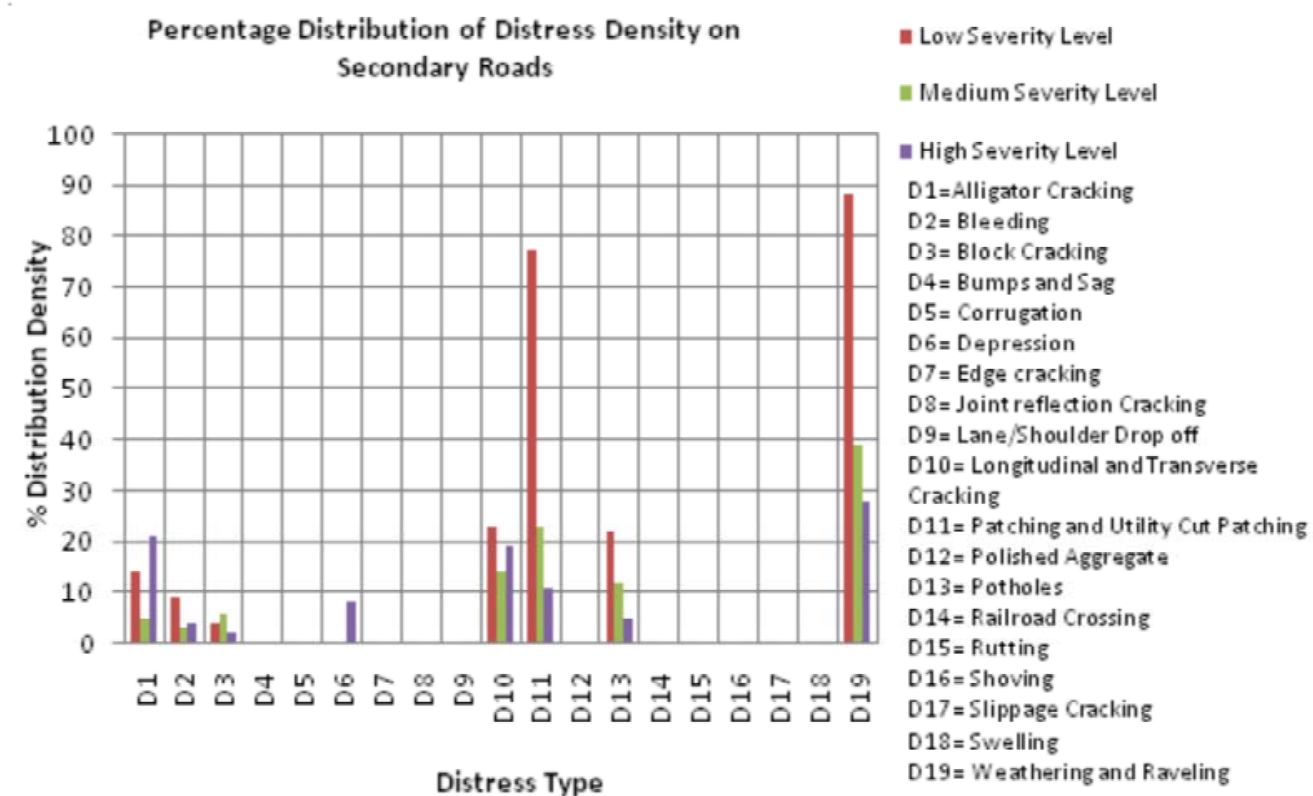

Figure 4. Percentage distribution of distress density on secondary roads.

From this figure, it is clear that $66 \%$ of the distresses found on the main roads network at a low severity level is patching and utility cut patching (D11). Types of longitudinal and transverse cracking (D10) represent about $55 \%$ of the distress types found at the lowseverity level. Other distress types found at low severity level include polished aggregate (D12), weathering and raveling (D19), rutting (D15), alligator cracking (D1), depression (D6), and bleeding (D2).

About $42 \%$ of the distress types at the medium severity level are longitudinal and transverse cracking (D10). Another $22 \%$ of the distress types at the medium severity level are the patching and utility cut patching (D11). Other distress types found at the medium severity level were polished aggregate (D12), weathering and raveling (D19), rutting (D15), block cracking (D3), alligator cracking (D1), bleeding (D2), and depression (D6).

About $21 \%$ of the distress types at high severity level are the alligator cracking (D1) type. Longitudinal and transverse cracking (D10) type represents $(18 \%)$ and polished aggregate (D12) account for about $(14 \%)$ of the total high severity distress types. About (11\%) of total distress are the patching and utility cut patching (D11) types. Rutting (D15) type represents only $(7 \%)$ of the high severity distress types. Other distress types showed at high severity level were weathering and raveling (D19), bleeding (D2), and block cracking (D3).

\subsection{Common Distress on Secondary Roads}

The percentage of distribution of distress types found on secondary road networks at the three levels of severity are presented in Fig. 4. The analysis indicated that $88 \%$ of low severity distress types found on secondary roads is the D19 type. Among the distress types with medium severity, D19 represents about $38 \%$ of the total distress types found on secondary roads. D11 type represents about $22 \%$ of high severity level types of distress.

\section{Pavement Condition Evaluation}

\subsection{Evaluation of Main Roads Condition \\ Condition Evaluation for Individual Main Roads}

The PCI for each main road out of the 11 selected main roads is shown in Fig. 5 bar graph. This figure shows that the average PCI for the selected main roads ranged from 60 to 91 , which reflects a rating of good to excellent. The minimum PCI value for all the sample units included in the analysis was found to be 19 , with a condition rating of very poor.

\section{Condition Evaluation for all Main Roads}

The PCI for the main road network were computed by averaging the PCIs for the selected roads because all the sample units were of the same size. A total of 22,700 meters of main roads representing 227 sample units was included. The average PCI for the main roads was found to be 76 , which indicates a general condition rating of very good. The analysis also indicated that $15 \%$ of Jazan's main roads have an average condition rating of excellent. The finding was that $65 \%, 15 \%$, and $5 \%$ of the networks were very good, good, and fair, respectively as indicated in Fig. 6. This good rating reflects the city's newly developed network. It also shows the municipality's great challenge of preserving the network and controlling its rate of deterioration. 


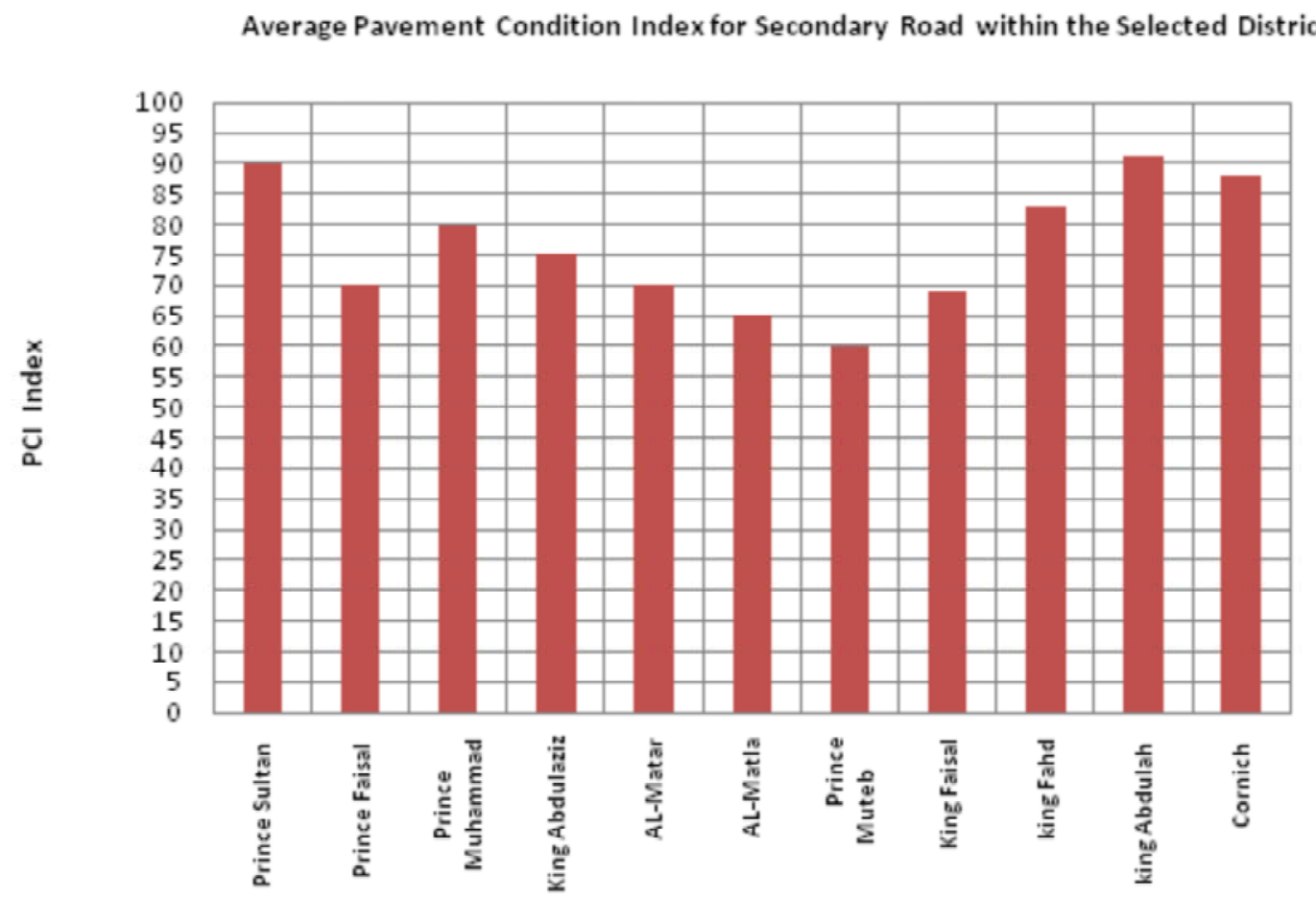

District Name

Figure 5. Average pavement condition index for the selected on main roads.

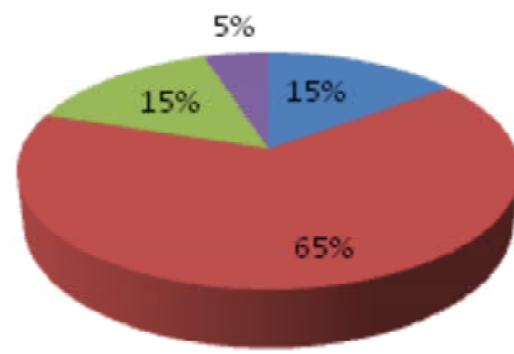

\author{
Excellent \\ - Very Good \\ Good \\ Eair
}

Figure 6. Average conditions rating for Jazan main road network.

\subsection{Evaluation of Secondary Roads Condition \\ Condition Evaluation within Each District}

The results of pavement condition for the secondary roads within each region are presented in Fig. 7 . The selected regions have been chosen from the north, south, east, west, and centre of the city. The figure shows that the average PCI for the selected regions range from $25-85$, with a general condition rating of poor to very good.

\section{Condition Evaluation for all Regions}

The pavement condition rating of all secondary roads was computed by averaging the PCIs of secondary roads within each region. A total area of 769,000 square meters of secondary roads representing 500 sample units within the selected 10 regions was con- sidered. The average PCI for the secondary road network of Jazan City was found to be 66 , which indicates a general rating condition of good. The percentage distribution of the condition rating of the secondary road network is presented in Fig. 8, which shows that about $65 \%$ of the secondary road network is in a critical stage and, if these roads are not properly maintained, they will deteriorate with time, and significant cost will have to be incurred to make repairs. A welldesigned pavement is likely to stay in good condition for a long time before it reaches what PAVER refers to as the "critical condition". The length of time it takes for the pavement to reach critical condition is the pavement life. Pavement life can be significantly increased by performing preventative maintenance. On the other hand, once the pavement reaches critical 


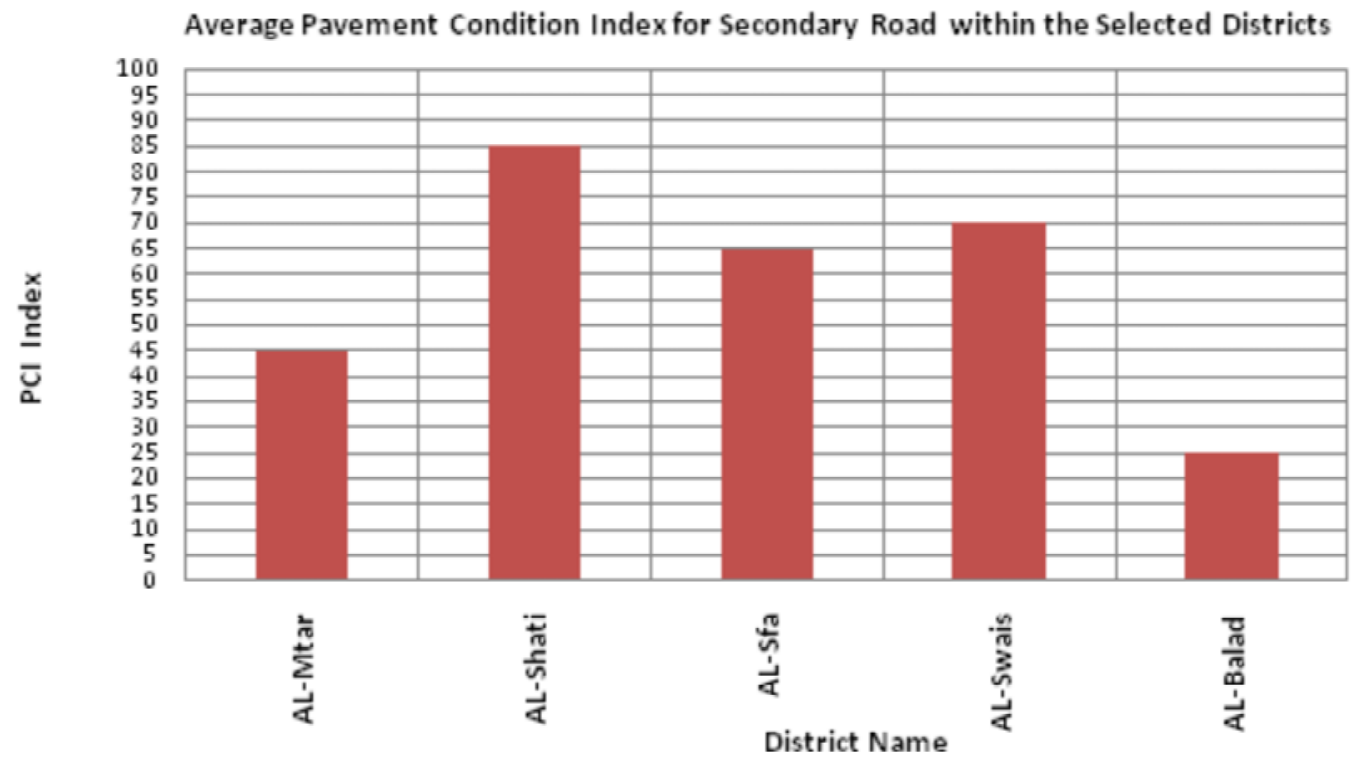

Figure 7. Average pavement condition index for the selected on secondary roads within the selected regions.

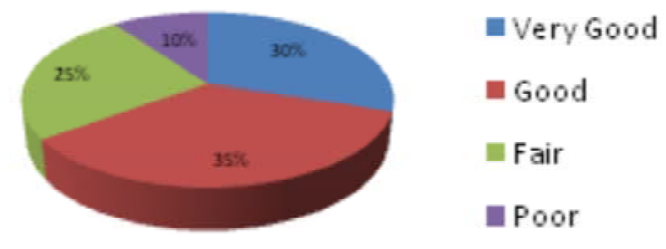

Figure 8. Average rating for Jazan secondary roads network.

condition, its rate of deterioration significantly increases and the return on investment of preventive maintenance decreases. For example, consider the question of the effectiveness of crack sealing if the pavement already has potholes. Therefore, critical PCI is the PCI value at which the rate of PCI loss increases with time. Usually this value ranges from 70 to 55 .

Additionally, Fig. 8 shows that $10 \%$ of the secondary network needs to receive a thick overlay. One of the foremost reasons for using a condition indices pavement management system is to trigger treatments (Deighton and Sztraka 1995). During a PMS analysis, a list of maintenance and rehabilitation strategies is generated. In generating this list, it is important that only feasible treatments are considered; otherwise the list will be impossibly long. To include only feasible treatments on the list, the PMS needs to know when a treatment is feasible and when it is not.

The process used by most PMS can be described as a simple decision process, where decision trees or trig gers are used. The major inputs to this process are the condition indices. For example, with a treatment such as a thick overlay, a condition index is needed to indicate when a road is in a condition that makes applying a thick overlay feasible.

Feasibility can be examined from an operational and economical perspective, and it is important not to confuse them. From an operational point of view, a thin $(25 \mathrm{~mm})$ overlay is sometimes impossible to actually place on the pavement. Consider a road with ruts, distortions, and severe roughness. If a PMS included a thin overlay treatment on the list of strategies for that pavement section, it would lose credibility.

Therefore, from an operational perspective, the PMS needs condition indices to indicate when a road is outside the operationally feasible zone of receiving a particular treatment. Usually, agencies begin their PMS by mimicking current practices; thus, this usually defines what is feasible from an operational point of view. In a study by Mubaraki (2012), the thick overlay $(50 \mathrm{~mm})$ which is the structural overlay is highly recommended when the PCI is less than 40 . The feasibility of its application should be based on developing pavement conditions, a model, and an application of that model in setting maintenance strategies at the project level for low volume urban roads (secondary roads). In this paper, it was found that $10 \%$ of the secondary roads in the Jazan City network had an AS PCI value below 35. Therefore, the view of feasibility treatment is important here because of two perspective criteria operations and economics (limited resources). 


\section{Conclusions}

Based on the results obtained from this study, the following may be concluded:

- The types of distress that commonly exist in Jazan City's main road network are patching and utility cut patching, longitudinal and transverse cracking, polished aggregate, weathering and raveling, and alligator cracking.

- The most common types of distress that exist on Jazan City's secondary road network are weathering and raveling, patching and utility cut patching, longitudinal and transverse cracking, potholes, and alligator cracking.

- The average pavement condition rating of Jazan's main road network is very good.

- The percentage of distribution of the pavement condition rating in Jazan City's main road network is $5 \%$ (fair), $15 \%$ (good), $65 \%$ (very good), and 15\% (excellent).

- About 20\% of Jazan's secondary road network needs immediate major maintenance such as thin and thick overlay.

- Jazan City's main road network is newly developed and, relatively speaking, in very good condition. Extensive effort should be made in order to preserve and improve the network. However, on the other hand, Jazan's secondary road network is in bad condition and needs immediate action taken to improve its condition.

\section{Acknowledgment}

The author would like to acknowledge the assistance he received from the staff of Jazan Municipality. Their assistance and support made the production of this paper possible.

\section{References}

Al-Swailmi S (1994), Framework for municipal maintenance management systems. Transportation Research Board, Transportation Research Board, TRR 1442, 3-10, Washington DC, USA.

Annual Book of ASTM Standards (2011), ASTM D5340-11, Standard practice for roads and parking lots pavement condition index surveys 04:(03).

Baladi GY, Snyder MB (1992), Highway pavements, student workbook publication No. FHWA HI-90026 Federal Highway Administration. National Highway Institute Course No.13114.

Deighton R, Sztraka J (1995), Pavement condition. dTV Technical Guide (Vol. 3), published by Deighton Associates Limited.

Haas R, Hudson WR, Zaniewski J (1994), Modern pavement management. Krieger Publishing Company, Malabor, Florida.

Mubaraki M (2004), Modeling the common pavement distress types on Riyadh streets Network. Master's thesis, King Saud University, Saudi Arabia.

Mubaraki M (2010), Predicting deterioration for the Saudi Arabian urban road network. PhD thesis, University of Nottingham, UK.

Mubaraki M (2012), Maintenance strategies at project level for low volume urban roads. International Journal of Pavement Research and Technology 5(4):225-233.

Shahin MY (2005), Pavement for airports, roads, and parking lots. Springer, New York, USA.

Shahin MY, Walteher J (1990), Pavement maintenance management for roads streets using the PAVER system. Technical Report M-90, U.S. Army Construction Engineering Lab. 